\title{
Using UNAIDS's organizing framework to assess Nigeria's national HIV monitoring and evaluation system
}

\author{
Kayode Ogungbemi ${ }^{1}$, Kola A. Oyediran ${ }^{2 *}$, Stephanie Mullen ${ }^{2}$, Anne LaFond $^{2}$, Aderemi Azeez $^{3}$, \\ David Boone $^{2}$, Tendayi Ndori Mharadze ${ }^{4}$, Natasha Kanagat ${ }^{2}$, Akinyemi Atobatele $^{5}$ \\ ${ }^{1}$ Strategic Knowledge Management, National Agency for the Control of AIDS (NACA), Abuja, Nigeria \\ ${ }^{2}$ MEASURE Evaluation Project, John Snow, Inc., Washington DC, USA; *Corresponding Author: koyediran@jsi.com \\ ${ }^{3}$ National AIDS and STIs Control Programme, Federal Ministry of Health, Abuja, Nigeria \\ ${ }^{4}$ Monitoring and Evaluation, Zimbabwe AIDS Network, Harare, Zimbabwe \\ ${ }^{5}$ United States Agency for International Development/Nigeria, Abuja, Nigeria
}

Received 8 February 2012; revised 28 March 2012; accepted 16 April 2012

\section{ABSTRACT}

The Nigeria National Response Management Information System (NNRIMS), developed in 2004 as a framework for monitoring and evaluating the country's response to HIV, does not function at an optimum level due to several challenges, including a bewildering proliferation of vertical reporting systems, competition among sectors, and the nascent nature of the monitoring and evaluation (M\&E) sub-systems within many institutions. An assessment of the existing M\&E system was conducted to verify whether the system has the capacities to provide essential data for monitoring the epidemic and identifying critical programming gaps. Nigeria's National Agency for the Control of AIDS (NACA) used an organizing framework for a national HIV M\&E system developed by UNAIDS, to assess the strengths and weaknesses of the NNRIMS to generate data for evidence-based decisionmaking. The participatory approach used during an assessment workshop ensured that the process was country-led and -owned to build consensus and local capacity, and that it encouraged adoption of a single national-level multisectoral HIV M\&E system. The assessment found an operable M\&E system at the national level but a much weaker system at the state and local levels and across seven other sectors. There are multiple data collection and reporting tools at the facility level that lead to vertical reporting systems, which increases the burden of reporting at lower levels, especially by service providers. Human resources are being developed, but problems remain with the quantity and qual- ity of staff. Data use, though evident at the national level, is still very weak among five of the seven sectors assessed. The assessment results have been used to develop a national costed M\&E workplan to which all stakeholders contributed in a coordinated response to strengthen the system.

Keywords: HIVIAIDS; Nigeria; 12 Components; M\&E System

\section{INTRODUCTION}

In 2005, development partners and governments worldwide agreed on the Three Ones [1] principles to manage the HIV response at the global and country levels: one agreed HIV/AIDS Action Framework that provides the basis for coordinating the work of all partners, one national AIDS coordinating authority with a broad-based multi-sector mandate, and one agreed country-level monitoring and evaluation (M\&E) system. Efforts at developing the third principle have accelerated globally. Governments and their partners began to focus on strengthening all HIV M\&E systems to capture data about their national HIV response and to measure achievements.

Two years later, this led to an international agreement on an organizing framework to make national HIV M\&E systems fully functional and to develop a single tool to assess them [2]. This framework helps stakeholders reach agreement on the performance goals of the system, assess system capacity, develop a capacity-building strategy and costed workplan, and introduce measures to monitor M\&E system performance over time. The related assessment tool-known formally as the 12 Components Monitoring and Evaluation System Strengthen- 
ing Tool-is used to guide the process of country-owned and -led self-assessment of the national or sub-national M\&E system using the 12 components necessary to build a functional M\&E system.

The Organizing Framework for a Functional National HIV M\&E System [2] describes these 12 components, as well as key performance elements for each component that can be used to assess progress and effectiveness. In Figure 1, the 12 components of an organizing framework for a functional national HIV M\&E system are illustrated as intersecting and interdependent parts of a larger national system.

The related guidelines based on the organizing framework for a functional national HIV monitoring and evaluation system support a holistic approach to system strengthening that uses the 12 components tool [4], as part of a three-day stakeholder workshop in November 2009 at the national level to assess the status of the 12 components within the national HIV M\&E system. In Nigeria this national system includes both information systems and sub-systems, as well as the organizations and individuals that coordinate and manage those systems: the National Agency for the Control of AIDS (NACA), Federal Ministry of Health (FMOH), umbrella organizations, other public sector ministries, and decentralized HIV coordination units (e.g., state and local offices) at health facilities and within the NGO sector.

In national HIV/AIDS programs, policymaking, program improvement, and accountability depend on the collection, analysis, reporting, and use of good-quality data. In Nigeria, both the human capacity and information infrastructure of the national HIV M\&E system are weak. Currently, the system cannot guarantee timely access to and analysis of high-quality data for reporting on core indicators [5]. The gaps-particularly in the Health Information System (HIS) and in information and communications technology (ICT) - must be addressed to ensure a fully functional and effective national HIV M\&E system.

Nigeria’s National Response Information Management

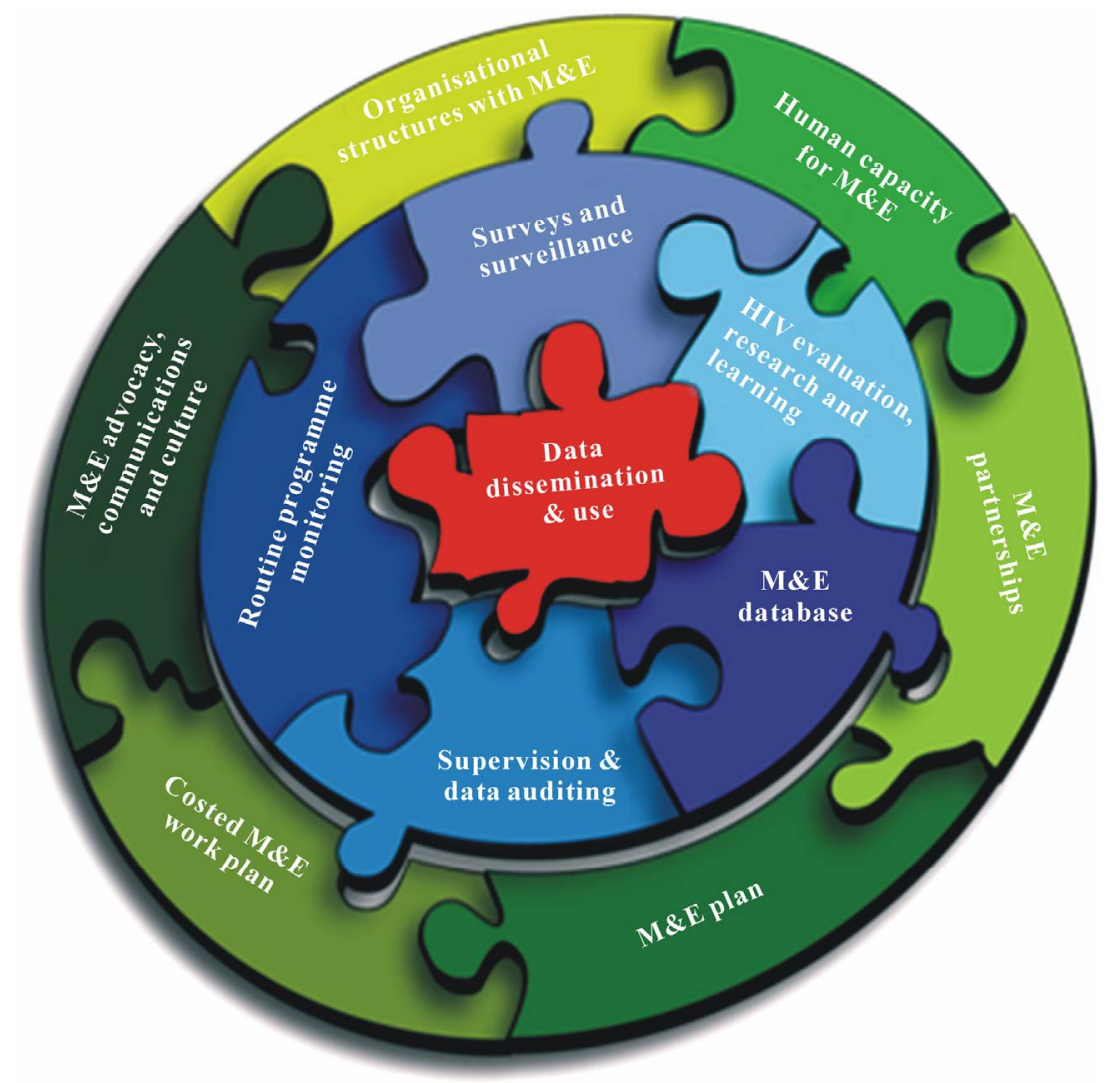

Figure 1. Courtesy of UNAIDS. The 12 components can be sub-divided and arranged into three linked resource and activity rings [3]: 1) The outer ring (green): This links 6 components related to people, partnerships, and planning that support data production and data use (i.e., the enabling environment for HIV M\&E to function). 2) The middle ring (blue): This links 5 components related to data management processes: collection, capture, and verification of all types of HIV M\&E data. 3) The inner ring (red): This involves analyzing data to create information, which is then disseminated to inform and empower decisionmaking at all levels. 
System (NNRIMS) has the mandate to collect information on multisectoral HIV/AIDS services, including community-level activities; strengthening NNRIMS and the M\&E system is a priority for the National Agency for the Control of AIDS (NACA) and other HIV/AIDS program stakeholders. But this task is made difficult by the proliferation of autonomous or semi-autonomous M\&E subsystems throughout the country's network of HIV/AIDS programs, which are mostly donor driven and are poorly harmonized with NNRIMS. Each program area—such as orphans and vulnerable children (OVC), antiretroviral therapy (ART), and prevention of mother-to-child transmission (PMTCT) - has its own M\&E sub-system that responds to the needs of funders rather than national M\&E efforts.

Another critical issue is the limited use of the NNRIMS platform by the private sector, especially private forprofit entities, to submit information [6,7]. Another challenge affecting the functionality of NNRIMS is low capacity at the sub-national level (state, Local Governmental Authority [LGA], and service delivery points) to manage M\&E systems, including the capacity to ensure good data quality and to use information routinely for decisionmaking.

NACA expressed an interest in carrying out an assessment of the national HIV/AIDS M\&E system and in developing interventions to address any gaps identified by the assessment. The assessment included all stake holders with significant roles and responsibilities in designing, planning, and managing the HIV/AIDS M\&E system. MEASURE Evaluation-a global technical assistance project supported by the United States Agency for International Development (USAID)—provided technical assistance and helped the Government of Nigeria implement the assessment in collaboration with other stakeholders, particularly UNAIDS.

\section{METHODS}

The M\&E assessment used a participatory and qualitative approach that allowed for discussion, reflection, and consensus building. The main activity of the assessment process was the completion of the 12 components tool by stakeholders to serve as a springboard for discussion and strategic planning and help build commitment to improving M\&E system performance. NACA led the assessment process using the 12 components organizing framework and tool [8]. The assessment employed three critical steps: the pre-assessment desk review, consultation with key stakeholders, and the stakeholders' M\&E assessment workshop.

The first step involved reviewing relevant documents related to the national HIV response in general and the multisectoral HIV M\&E system in particular, including the National Policy on HIV and AIDS, the National Strategic Plan on HIV/AIDS, the Nigerian National Response Information Management System Operational Plan [9], and data collection and reporting tools for both facility- and non-facility-based service delivery and related guidelines.

The second step was a consultation with a small group of key stakeholders to clarify the purpose of the M\&E assessment, the facilitation processes and logistics for the workshop, and the critical next steps that would follow the assessment. The meetings allowed these stakeholders to interject country-specific requirements and needs into the assessment process, particularly those related to the review of the current National Strategic Framework (NSF) (2005-2009) and the development of the next NSF (2010-2016). This group of stakeholders included government institutions and partners working with the Government of Nigeria-including the M\&E Assessment Steering Committee, United States Government (USG) missions, and the UNAIDS M\&E advisor-to mitigate the impact of HIV/AIDS and implement the HIV/AIDS M\&E system.

The third step was the three-day M\&E Assessment Workshop held in Kaduna, Nigeria, from November 2 to 4, 2009. The workshop proceedings were guided by the 12 Components M\&E System Assessment Guide [10]. Participants were drawn from all the sectors that contribute to the national HIV response and divided into seven stakeholder groups: NACA, the Ministry of Health, other line ministries, civil society umbrella organizations, subnational level agencies responsible for coordinating HIV/ AIDS responses, health facilities, and other implementers of AIDS programs. Steering Committee members served as facilitators during the workshop, guiding group discussions and leading participants in the completion of group worksheets. Each stakeholder group was supported by at least two facilitators.

The three-day workshop began with an update on the drafting of the national strategic framework, a brief introduction to the 12 components organizing framework, and an orientation to the tool. The participants in the seven stakeholder groups went through the assessment checklist, deliberating on the statements and coming to consensus on appropriate responses. At the end of each component, they drafted action points for strengthening weaknesses and addressing gaps identified within each component.

After the assessment, NACA identified a core team of stakeholders to act on the assessment results by developing a costed national M\&E workplan by the end of 2009 .

\section{RESULTS}

The overall results of the assessment appear below by 
component in Table 1. Figure 2 shows how stakeholders from NACA ranked themselves according to the 12 components.

\section{DISCUSSION}

The assessment results confirm that the coordinating agencies at the national level have organizational structures that help them perform their M\&E mandates and functions, but these structures do not exist at the subnational, civil society, and facility levels. There is also a need to employ skilled personnel within the organiza- tional structure to operate the system. This requires organograms, job descriptions based on approved and costed workplans, and other organizational tools that identify required or existing skilled staff, such as epidemiologists, in order for NACA to effectively and responsibly perform according to its M\&E mandates.

The results also suggest that some attempts have been made in the past to enhance the skills of individuals operating the HIV/AIDS M\&E system at various levels, from the national to the sub-national. However, concerted efforts are needed to improve capacity building in a holistic way that focuses on individuals, organizations,

Table 1. Workshop results summary.

\begin{tabular}{|c|c|}
\hline Component & Result \\
\hline $\begin{array}{l}\text { 1) Organizational } \\
\text { structures }\end{array}$ & $\begin{array}{l}\text { National-level entities (e.g., NACA, FMOH-NASCP, umbrella organizations, and federal ministries and departments), } \\
\text { have established organizational structures. However, lower-level entities (e.g., states and LGA), health facilities, civil } \\
\text { society organizations, and other implementing partners have very poor structures and, in some instances, are not even } \\
\text { aware of their M\&E mandate. }\end{array}$ \\
\hline 2) Human capacity & $\begin{array}{l}\text { The weakness of this component may be attributed to the nature of human capacity development in an ever-changing } \\
\text { work environment, as well as a general lack of skills to handle the newly harmonized HIV M\&E systems. Nigeria } \\
\text { understands the need to invest in human capacity development for HIV M\&E and is currently working to build a } \\
\text { team of highly motivated M\&E professionals. }\end{array}$ \\
\hline 3) Partnerships & $\begin{array}{l}\text { Partnerships for HIV M\&E are being built and maintained. However, limited success has been registered at the state } \\
\text { level. This is a critical gap, since actual implementation and the sources of data are at the lower levels. }\end{array}$ \\
\hline 4) M\&E planning & $\begin{array}{l}\text { Only the national HIV M\&E plan was assessed in this component. The plan complies with international standards and } \\
\text { is aligned with the NSF; however, it was developed before a number of sector plans had been developed and, therefore, } \\
\text { linkages are poor. There is room to improve the national M\&E plan as a new generation plan to monitor and evaluate } \\
\text { NSF is being developed. }\end{array}$ \\
\hline 5) Costed M\&E workplan & Nigeria had not developed a national multi-partner costed M\&E workplan. \\
\hline $\begin{array}{l}\text { 6) Advocacy, } \\
\text { communication, and } \\
\text { culture }\end{array}$ & $\begin{array}{l}\text { While advocacy for HIV M\&E is necessary, it is clear that consciousness of the need for HIV M\&E is growing among } \\
\text { all sectors, in particular at NACA, FMOH, other federal ministries, and implementing partners. However, this is not } \\
\text { evident at the state level and within civil society umbrella organizations. At the facility level and among other } \\
\text { implementers, M\&E is donor driven rather than driven by organizational culture. }\end{array}$ \\
\hline 7) Routine monitoring & $\begin{array}{l}\text { Health programs have a stronger routine program monitoring system than such non-health programs as orphans and } \\
\text { vulnerable children (OVC) and home-based care. There are national data collection guidelines for health-related } \\
\text { programs such as antiretroviral therapy, PMTCT, HIV testing and counseling, HIV/tuberculosis, and OVC. Although } \\
\text { guidelines exist for private sector health facilities, enforcement of adherence to these guidelines poses a challenge. }\end{array}$ \\
\hline $\begin{array}{l}\text { 8) Surveys and } \\
\text { surveillance }\end{array}$ & $\begin{array}{l}\text { Nigeria has a good system for surveys and surveillance that provides critical information for the HIV response. } \\
\text { However, the scope of the surveys and surveillance is limited to providing estimates at the national level. There are } \\
\text { few inferences that states can make from the data that are generated; thus states rarely use these data to inform their } \\
\text { own decisionmaking and programming. }\end{array}$ \\
\hline 9) HIV databases & $\begin{array}{l}\text { While Nigeria has a number of HIV databases that capture, verify, analyze, and present program monitoring data, } \\
\text { these are not linked to each other. There is thus a high likelihood of duplication of effort and poor resource use. }\end{array}$ \\
\hline $\begin{array}{l}\text { 10) Supportive supervision } \\
\text { and data auditing }\end{array}$ & $\begin{array}{l}\text { Nigeria lacks these components because most M\&E-related processes are donor driven. NACA needs to develop the } \\
\text { required guidelines for data auditing and supportive supervision, as well as schedule the exercises in its annual } \\
\text { workplan and conduct them as planned. }\end{array}$ \\
\hline $\begin{array}{l}\text { 11) Evaluation and } \\
\text { research }\end{array}$ & $\begin{array}{l}\text { Various HIV-related evaluation and research studies have been conducted in Nigeria; NACA had recently made some } \\
\text { efforts to coordinate the HIV research and evaluation studies, but very little ground had been covered. Nigeria had } \\
\text { done relatively well in conducting joint reviews of the national response. }\end{array}$ \\
\hline $\begin{array}{l}\text { 12) Data dissemination } \\
\text { and use }\end{array}$ & $\begin{array}{l}\text { Most sectors produce written reports on their activities, but only NACA disseminates the information through its } \\
\text { website to the various stakeholders and the public. Some stakeholders reported a lack of awareness of NACA's } \\
\text { information products. There is evidence of M\&E information use in the review and development of the national } \\
\text { strategic framework. However, Nigeria has not conducted a stakeholder information needs assessment at national and } \\
\text { sub-national levels to determine the nature of information required for decisionmaking. Meanwhile, data use is very } \\
\text { limited at the sub-national level, and information products are not disseminated to the various stakeholders. }\end{array}$ \\
\hline
\end{tabular}




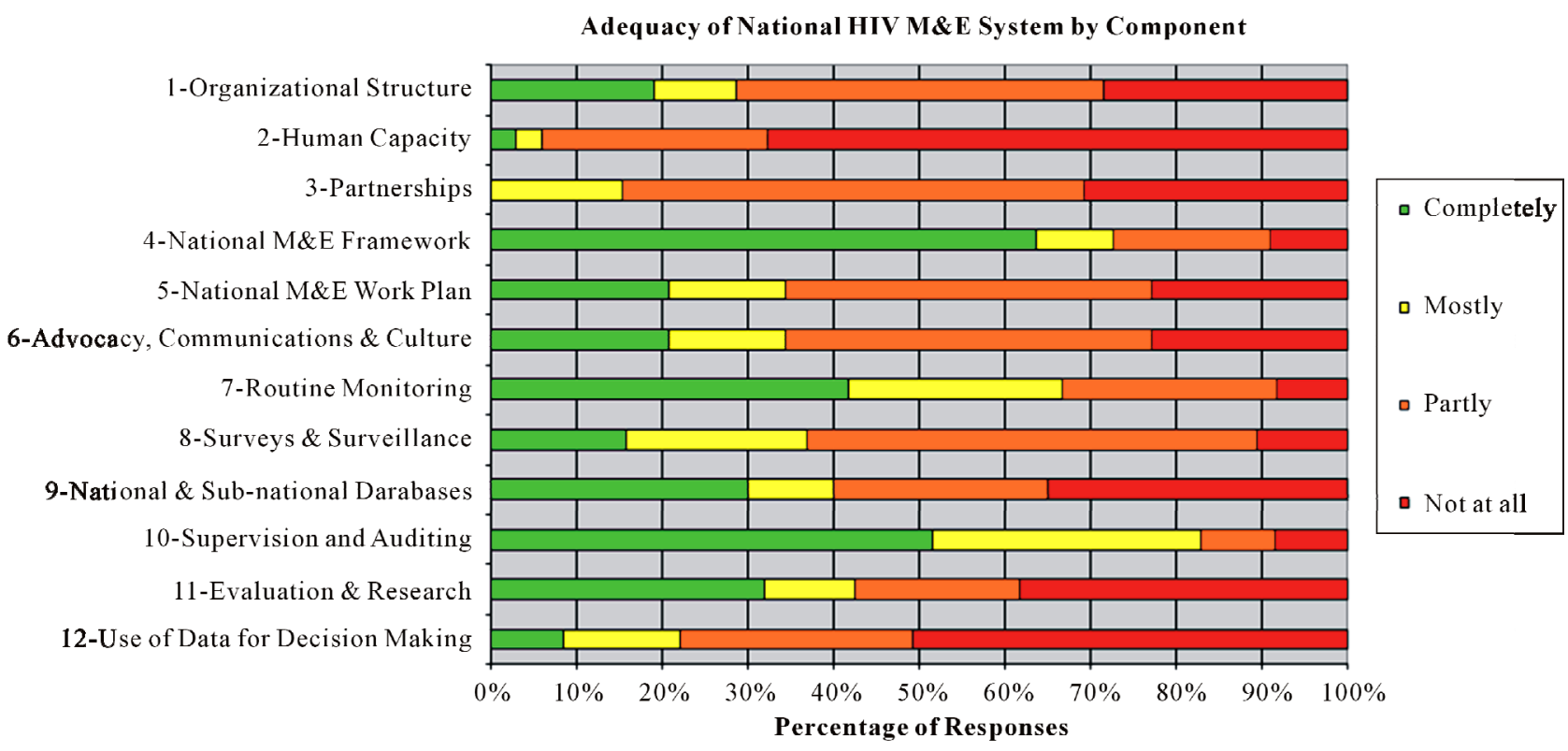

Figure 2. Dashboard showing NACA's performance on 12 components [8].

and systems. A capacity building plan should be required to address the capacity of the individuals, organizations, and systems at the national, sub-national, and service delivery levels involved to execute HIV M\&E functions or manage employees doing this work.

Those involved in the country's HIV M\&E system do not come from the same organization or sector. To bring these stakeholders together, the Nigerian Government established and inaugurated a national M\&E Technical Working Group (TWG) in line with the principle of the "Three Ones". The TWG provides technical and administrative direction to implement the NNRIMS framework (although the sub-committees have not carried out their charters effectively). The TWG also promotes strong partnerships among the diverse people from different organizations toward successful implementation of the NNRIMS. However, weak organizational structure and limited human capacity affect the functionality of the state- and sector-level M\&E TWGs. The assessment further reveals that the charters and memberships of subcommittees are not based on the comparative advantage of the organization or individual representing the organization. Correcting this would maximize the benefit from these partnerships for planning, coordination, and management of the multisectoral HIV M\&E system.

This assessment shows that a national M\&E plan together with a costed workplan are the key ingredients for a functional M\&E system that can produce high-quality data for evidence-based decisions. Both are designed to describe the principles and functions of the M\&E system, the data the system will collect, and details of how the system will operate. In 2006, Nigeria developed a national HIV/AIDS M\&E plan through a participatory process with the majority of the sectors, but these did not translate into sub-national M\&E plans. One reason is that the M\&E plan was not supported with a costed M\&E workplan, which contributed to duplication of efforts and inefficient use of human and financial resources that could have been used to strengthen the HIV M\&E system. A costed, multi-year, multisectoral, and multilevel workplan would have avoided some of these inefficiencies and helped to prioritize, plan, and coordinate M\&E system activities. The assessment also revealed that there is no guidance on how to define, produce, and share information products resulting from planned M\&E activities, due to the lack of M\&E champions among HIV managers, planners, and policymakers.

It was evident from the assessment that health programs have a stronger routine program monitoring system than non-health programs, perhaps because the government and donor agencies pay more attention to healthrelated services. Routine program monitoring is still beset by such challenges as the proliferation of data collection and reporting tools, a lack of country ownership, poor data quality, a lack of guidelines and protocols, and poor supportive supervision for those at the lower level. Nigeria has a good HIV surveillance system and conducts surveys and evaluations on a regular basis that provide data for most outcome and impact indicators in the M\&E plan. However, the sample size of most surveys, surveillance studies, and evaluation studies has not been sufficient to provide estimates at sub-national levels, so policymakers at those levels do not know whether programs are reducing the impact of HIV in their states.

The assessment shows that many databases are available throughout Nigeria for collecting, capturing, and 
verifying the data needed for the HIV M\&E system. However, most are donor driven and lack interoperability; they also present a burden to those operating them at lower levels, especially when there are two or more donors that each has its own data reporting system. For instance, at the time of the assessment, NACA was using two databases, but the interoperability of the two systems remains unknown. Finally, the overriding purpose of the $M \& E$ system is to generate data to create information for decisionmaking, but data use is limited among the stakeholders, particularly at the sub-national levels and service delivery points. This could be due to the fact that most stakeholders at the sub-national level regard the M\&E system as a reporting requirement only for the donor or funders of the programs, not for the Nigerian Government.

The key intent of the assessment was to determine the strengths and weaknesses of Nigeria's current M\&E system for tracking the performance of the country's HIV/ AIDS response. After the completion of the 12 components tool and review of the key findings, NACA and other stakeholders set up a committee to develop a strategy for implementing key assessment recommendations and a costed M\&E workplan. The implementation strategy was then presented to the National M\&E TWG for its approval. The assessment results have been used to develop a national costed M\&E workplan-to which all stakeholders contributed-to ensure a coordinated response for strengthening the system. NACA initiated the development of the workplan with a five-day stakeholders' consensus-building workshop in March 2010. Participants identified top-priority activities for 2010 and 2011, created a budget to implement the activities, named the organizations responsible for implementation, and issued a timeline.

The results also showed that there is proliferation of indicators and reporting tools throughout all sectors. One of the major strategic activities identified in the workplan is the harmonization of indicators and existing tools, which - in collaboration with other stakeholders-NACA has started to do, as well as to use a sustainable approach to developing key guidelines and protocols for supportive supervision and capacity building. The assessment results will also inform the development of an HIV/ AIDS research and evaluation agenda for the country.

\section{CONCLUSIONS}

These results have important programmatic and policy implications for the Government of Nigeria and NACA in particular. The factors affecting the functionality of HIV M\&E system are complex and multi-dimensional. For instance, the M\&E system is affected by the enabling environment needed for HIV M\&E to function: the peo- ple, partnerships, and planning that support data production and data use. Thus, data management processes should be properly addressed in order to have a single robust and responsive HIV M\&E system for the entire country.

There are many potential obstacles to achieving this goal, with the lack of resources-especially human resources - chief among them. The tasks that lie ahead require the cooperation of many agencies and players already dealing with the burden of developing and expanding HIV/AIDS response programs; for them, the development of M\&E systems is an additional and onerous responsibility. To overcome these challenges, a more systematic and long-term approach to the development of M\&E systems is needed for the sub-national sectors, as well as a strategy to track implementation. Each level of HIV operations should be part of the M\&E system, with indicators and measures and tools that are appropriate to its operations and consistent with national reporting categories. Systems for verifying reported data also need to be established. Staff at all funded projects need to undergo basic training in M\&E principles and practices. Policymakers and donors need to endorse the value of M\&E as both a reporting and program management practice to develop stronger buy-in of program personnel.

The assessment also revealed the need to develop much stronger supportive supervision, feedback loops, and technical assistance in M\&E at the sector and subnational levels, including network organizations and the private sector. The need for M\&E capacity development for HIV/AIDS service providers and for those at the government ministries, agencies and departments, subnational levels (states and LGAs) and umbrella organizations emerged from the assessment. Nigeria also needs an M\&E capacity building program that would include the following components: basic principles and practices of program evaluation, development of M\&E strategies and frameworks at the organizational level, development of indicators and measures, basic data capture and analysis, report writing, and alignment with national M\&E systems.

More specialized areas of M\&E also have unmet training needs for development and use of database software, cost-effectiveness analysis, assessment of the socioeconomic impact of HIV/AIDS, training in the use of geographic information systems (GIS) technology, advanced qualitative analysis, outcome and impact assessment, and M\&E of management systems and strategies, especially in human resources and governance (organizational development). Training initiatives need to be aligned with and supported by a national M\&E framework, and a process for developing consensus around a national set of core indicators needs to be implemented. It is also evident from the assessment that a national 
M\&E coordination framework should incorporate subnational coordination, a view strongly expressed by development partners.

Underlying all of this is the need for the development of a robust national health information system that links disease- or program-specific sub-systems for tracking program outcomes, captures and collates data, and facilitates financial management of programs. A national health strategy requires support from a health information system that contains data about serological surveillance, behavioral surveillance, coverage of essential services by state and sector, and the socioeconomic and population impact of the epidemic and its impact on social service sectors. This information system needs to be supported by a plan for information flow from program level to national level, as well as coordination of research and surveillance efforts, and should as far as possible allow for disaggregation of data to at least the state level to facilitate use of information by program managers.

\section{ACKNOWLEDGEMENTS}

The authors acknowledge the funding and technical supports provided by MEASURE Evaluation. MEASURE Evaluation is funded by the United States Agency for International Development (USAID) through Cooperative Agreement GHA-A-00-08-00003-00 and is implemented by the Carolina Population Center at the University of North Carolina at Chapel Hill, in partnership with Futures Group International, ICF International, John Snow, Inc., Management Sciences for Health, and Tulane University. The authors appreciate the editorial review by our colleagues at JSI (Margaret Dadian and Sarah Hiller) that has helped make the paper more concise. The comments and suggestions by the anonymous reviewer(s) are also recognized. The views expressed in this publication do not necessarily reflect the views of USAID or the United States Government but are solely those of the authors and do not represent the views of their institutions.

\section{REFERENCES}

[1] UNAIDS (2004) “Three Ones” key principles: Coordina- tion of national responses to HIV/AIDS-Guiding principles for national authorities and their partners. Conference paper \#1 from Washington consultation, Washington DC.

http://data.unaids.org/una-docs/three-ones_keyprinciples en.pdf

[2] UNAIDS Monitoring and Evaluation Reference Group (2008) Organizing framework for a functional national HIV M\&E system. UNAIDS, Geneva.

[3] UNAIDS Monitoring and Evaluation Reference Group. (2010) Guidance on capacity building for HIV monitoring and evaluation. UNAIDS, Geneva.

[4] UNAIDS Monitoring and Evaluation Reference Group (2010) 12 components monitoring \& evaluation system assessment guidelines to support preparation, implementation and follow-up activities. UNAIDS, Geneva.

[5] Mackay, K. (1999) Evaluation capacity development: A diagnostic guide and action framework. ECD Working Paper Series 6. World Bank Operations Evaluation Department, Washington DC.

[6] Nigeria Federal Ministry of Health (2008) Health management information system, monitoring and evaluation in Nigeria health sector. Abuja.

[7] Nigeria National Agency for the Control of AIDS (2010) Concept paper on harmonization of indicators, data collection and reporting tools for HIV/AIDS activities in Nigeria. Abuja.

[8] Mharadze, T.N., Ogungbemi, K., Boone, D. and Oyediran, K. (2010) Report on the status of the Nigerian national HIV monitoring and evaluation system: Assessment using 12 components system strengthening tool. MEASURE Evaluation, Chapel Hill.

[9] Nigeria National Agency for the Control of AIDS (2007) Nigerian national response information management system operational plan: HIV/AIDS guidelines for national M\&E plan. Abuja.

[10] UNAIDS (2010) 12 components M\&E system strengthening Tool. UNAIDS, Geneva. 